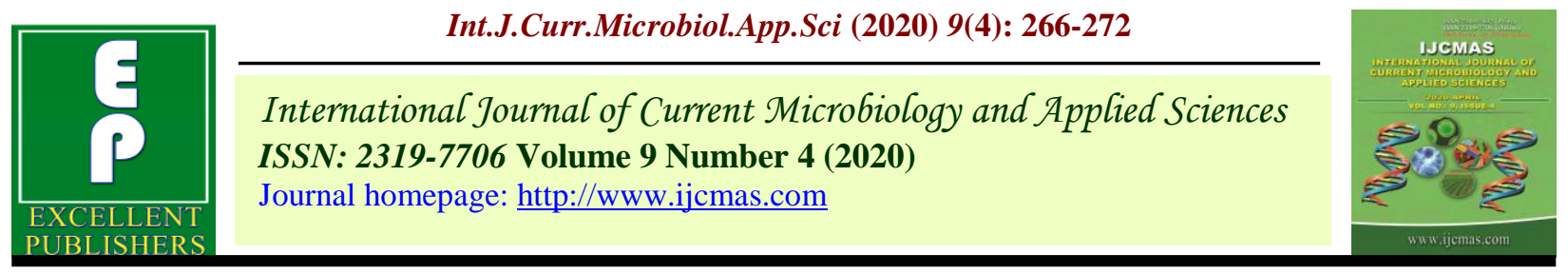

Original Research Article

https://doi.org/10.20546/ijcmas.2020.904.032

\title{
Prevalence of Non-responders among Medical Laboratory Technicians and Staff Nurses Evaluated for Anti Hepatitis B Surface Antigen Titre
}

\author{
V. Balamurali, R. Sujith*, K. V. Leela, A. Karthik and T. Jayaprakash \\ Department of Microbiology, SRM Medical College Hospital and Research Centre, \\ SRM Institute of Science and Technology, Kattankulathur - 603203, \\ Kancheepuram District, Tamilnadu, India \\ *Corresponding author
}

\section{A B S T R A C T}

Keywords

Antibody to

Hepatitis B surface

antigen, Hepatitis B

virus, Chemi

Luminescence

Immuno Assay,

Health care

workers, Non

responders,

Hepatitis B

Infection

Article Info

Accepted:

04 March 2020

Available Online:

10 April 2020
Hepatitis B Infection is an occupational high risk factor. Immune response for vaccination was measured by Antibody to Hepatitis B surface antigen (anti HBs) levels. A course of Hep B vaccine yields a protective immune response (anti-HBs $\geq 10 \mathrm{mIU} / \mathrm{mL}$ ) this study was designed to determine the anti HBs levels in Health Care Workers (HCWs) working as laboratory technician and staff nurses. The blood samples were collected from 546 HCWs, Anti HBs level was estimated using Chemi Luminescence Immuno Assay (CLIA) before and after 0,1,6 month of vaccination. Among 546 HCWs, before vaccination $83 \mathrm{HCWs}$ was non-protected, after $0^{\text {th }}$ dose of vaccination among $83 \mathrm{HCWs}, 25$ were again not protected. After administer two more doses, 4 were again non-protected. These $4 \mathrm{HCWs}$ was termed as "Non Responders", have confirmed there vaccination history of complete dose with evidence and has remained to be non-protective after the two sets of complete vaccination course administered.

\section{Introduction}

Hepatitis B infection (HBI) is a potentially life-threatening liver infection resulting from hepatitis B virus (HBV). Cirrhosis and liver cancer is the high risk for death and $\mathrm{HBV}$ also cause chronic infection (1). HBI is an occupational high risk infection for health care workers (HCWs) exposed to infected blood and contaminated needles and sharps
(2). In 2015 World Health Organization (WHO) estimates, chronic HBV infection was residing in 257 million individuals confirmed by hepatitis B surface antigen (HBsAg positive), in the same year 887 thousand deaths were reported worldwide, commonly due to cirrhosis and hepatocellular carcinoma (3).In India, prevalence of general population was $1.1 \%$ to $12.2 \%$ were HBsAg positive, Average prevalence was estimated that $3 \%$ - 
$4 \%$, due to chronic HBI approximately 40 million people were infected (4). Antibody to Hepatitis B surface antigen (anti-HBs) become a serological marker for both vaccine induced immunity and immunity because of infection. Protective immune response after vaccination as per $\mathrm{CDC}$ should be $\geq 10$ $\mathrm{mIU} / \mathrm{mL}$ (5). A hepatitis B vaccine "nonresponder" refers to an individual who does not induce Anti $\mathrm{Hbs}$ even after took two complete course of vaccination $(0,1,6$ months) and those with acute or chronic HBI has been excluded (6).

The main objectives of the study assess the prevalence of HBV non-responders among technician and staff nurses by evaluating protective Anti HBs in a tertiary care hospital in Kancheepuram district (South India), in a period of about twelve months, from January 2019 to January 2020.

The results of the study can be used to analyses the protective and non-protective group of HCWs before and after vaccination and to track the non-responders after two complete set of vaccination.

\section{Materials and Methods}

This was a Cross-sectional study conducted on $546 \mathrm{HCWs}$ who are in regular exposure to HBV infection. Institutional Ethics Committee has authorized this study (1520/IEC/2018 on 14.12.2018). The study group includes only Laboratory technicians and Staff nurses. Excluded groups were other HCWs other than staff nurses and laboratory technician and also excluding nursing students and short term internship individual in Laboratory. HCWs who had received blood component therapy like whole blood, plasma, immune globulin administration during the preceding months and immunosuppressed HCWs are also excluded. Previous vaccination status was obtained for all $\mathrm{HCWs}$ included in the study. Patient information sheet, consent form as been obtained duely signed by the HCWs participated in this study.A whole blood sample was collected with all aseptic precautions by a trained phlebotomist as per WHO guidelines(7) and allowed to clot and centrifuged to separate the serum. Anti-HBs titer was quantified in Chemiluminescence Immuno Assay (CLIA) method before and after 0, 1, 6 month vaccination. The Anti HBs titre of those showing > $10 \mathrm{mIU} / \mathrm{ml}$, was eventually excluded from the study.

\section{Results and Discussion}

Anti-HBs were quantified using Chemiluminescence Immuno Assay (CLIA) and results were recorded. Out of $546 \mathrm{HCWs}$, number of males and female were 85 (15.6\%), $461(84.4 \%)$ respectively. Among the total 546 HCWs, nurses constituted of $439(80.4 \%)$ and technicians were $107(19.6 \%)$ as shown in figure 1. All $546 \mathrm{HCWs}$ serum was tested for anti-HBs, result shows $463(84.8 \%)$ were Anti HBs protected, 83 (15.2\%) were Anti HBs non-protected as shown in figure 2. As per the reference guidelines, those who having anti $\mathrm{HBs} \geq 10 \mathrm{mIU} / \mathrm{mL}$ are termed as protected and those having $<10 \mathrm{mIU} / \mathrm{mL}$ are termed as non-protected.

Out of $83(15.2 \%)$ non-protected HCWs, in that $17(3.10 \%)$ technicians and $66(12.10 \%)$ staff nurses were $<10 \mathrm{mIU} / \mathrm{mL}$. Out of 83 non protected HCWs, 19 (3.46\%) had completed their course, $39(7.14 \%)$ had incomplete vaccination, 23 (4.21\%) HCWs don't know their vaccination status and $2(0.39 \%)$ were not vaccinated as shown in figure 3. After Administered $1^{\text {st }}$ dose of Hep B Vaccine for 83 (15.2\%) non-protected HCWs, 67 (12.25\%) were participated for Anti $\mathrm{HBs}$ titre test remaining 16 (2.95\%) were dropped out from the study since they left the job for which result shows $42(7.69 \%)$ were protected and 
$25(4.56 \%)$ were non-protected. After Administered $2^{\text {nd }}$ and $3^{\text {rd }}$ dose of Hep B Vaccine for 25 (4.56) non-protected HCWs again their Anti HBs titre was analyzed and that result shows $17(3.10 \%)$ were protected, 4 $(0.73 \%)$ were non-protected $4(0.73 \%)$ has again dropped out of my study. Out of 4 $(0.73 \%)$ non-protected HCWs, $1(0.18 \%)$ was technicians and $3(0.55 \%)$ were staff nurses

The total sample size of the study as 546 Health care Workers and among which $19.6 \%$ were technicians and $80.4 \%$ were Staff Nurses. $546 \mathrm{HCWs}$ included in the study we had $15.5 \%$ Male $(10.2 \%$ technicians, $5.3 \%$ staff nurses) and $84.5 \%$ female $(9.5 \%$ technicians, $75 \%$ staff nurses). This shows that in categories the percentage of female health care worker is employed more in nursing field, where as both male and female are employed equally as technicians. The number of $\mathrm{HCW}$ who had completed their course of vaccine was $3.46 \%, 7.14 \%$ had incomplete course, $4.21 \%$ did not know their vaccination status and $0.39 \%$ were not vaccinated. Both not vaccinated and didn't know their vaccination status comes under incomplete vaccination that shows total of 64 $(12.25 \%) \mathrm{HCWs}$ After administered $1^{\text {st }}$ and $6^{\text {th }}$ month doses of Hep B vaccine for nonprotected $25(4.56 \%)$ HCWs, $0.73 \%$ HCWs were dropped their jobs, $3.10 \% \mathrm{HCWs}$ were protected and $0.73 \%$ were non-protected.

The occupation based percentage of nonprotected healthcare workers are $0.55 \%$ Staff nurses, $0.18 \%$ technician. As per the CDC guidelines, the individuals who remain to have lower Anti HBs titre even after two series of complete vaccination are to be termed as non-responders. All those 4 $(0.73 \%)$ repeatedly non-protective category of health care workers in our study as termed as "Non-responders" have confirmed there vaccination history of complete dose with evidence and has remained to be nonprotective after the second set complete vaccination course administered by us. This study shows that HCWs specifically technicians and nurses has non-responder of $0.73 \%$ in a tertiary care hospital of Tamilnadu, India.

As stated by Amaddah radia et al., (8), Health employees constitute a population of chance for being exposed and getting transmission of the Hepatitis B Virus (HBV). Hence, raising a group of worker's attention and introducing compulsory HBV vaccination for all health care workers is important. In our study to signify the importance of HBV the study had free vaccination, screening and analysis of the immune response with the support of our institution with its hospital infection control committee.

In this study, total of 546 health care workers were suggesting they want for proactive implementation of HBV vaccination program. Considering on the Increasing age, time period and overweight could have decreased the immunity for those who have been previously vaccinated and for those who had remained to be as non-responders shall be the factors, as referred in a study by Praveena reddy et al., (9). As per the results of our study, the constant low titer of individuals after repeating a booster vaccination and complete course of vaccination emphasizes the want for monitoring vaccination reputation amongst health care workers.

The study have proved that there a gradual decline in antibody titers as duration of post vaccination increased. The percentage of subjects who have been non-protected after five and 10 years after vaccination were $20 \%$ and $27 \%$ respectively. 
Figure.1 Occupation wise distribution of technicians and staff nurses $(n=546)$

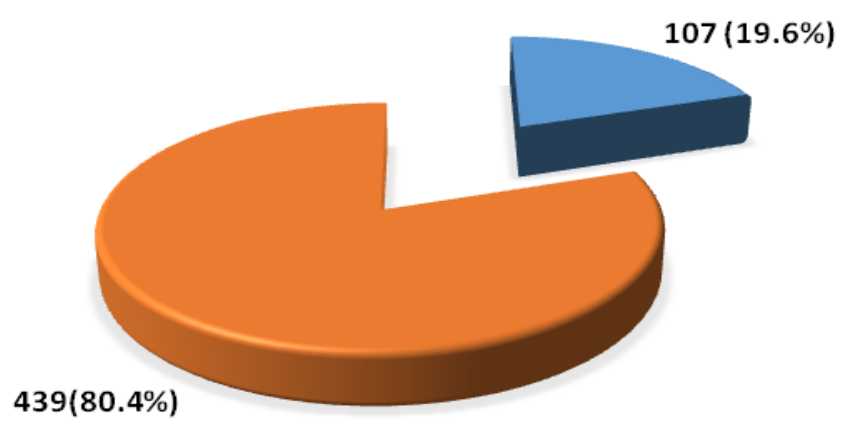

Technicians

Staff Nurses

Figure.2 Occupation wise distribution of healthcare care worker based on Anti-hbs titre $(\mathrm{n}=546)$

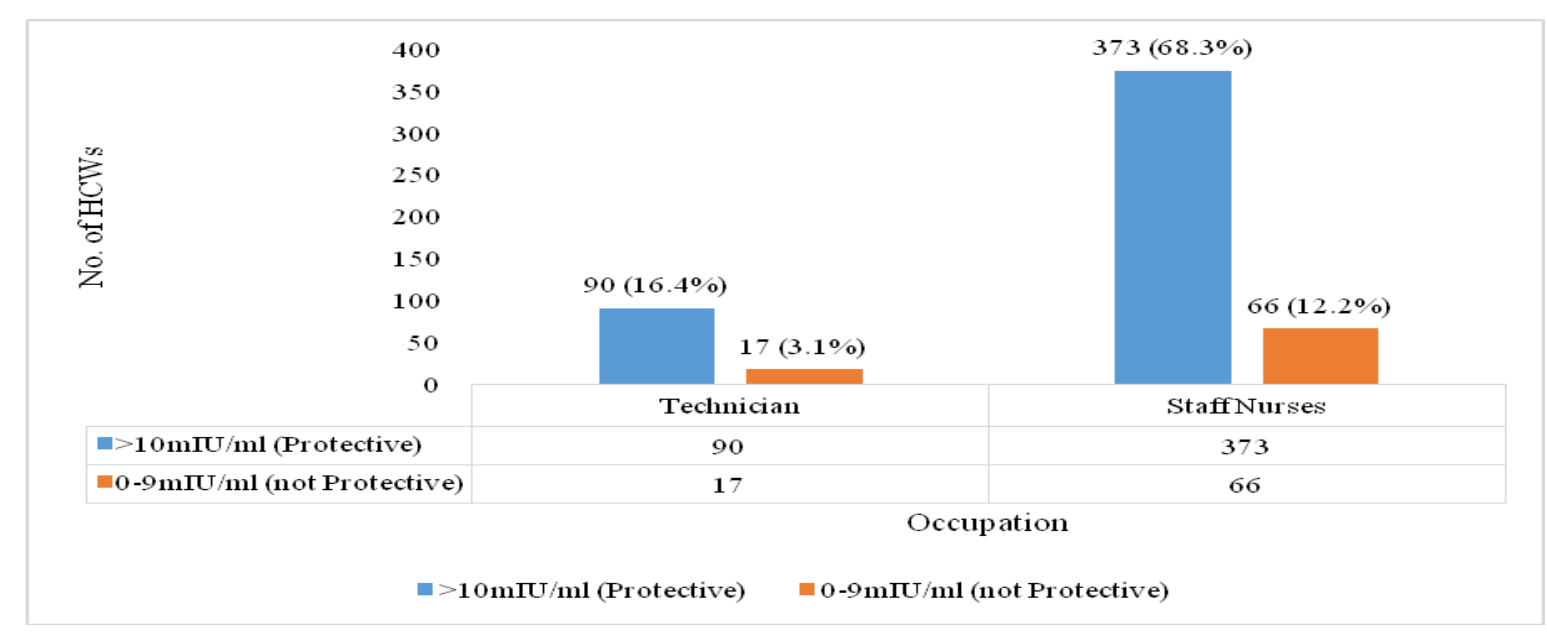

Figure.3 Initial vaccination status of health care workers $(n=83)$

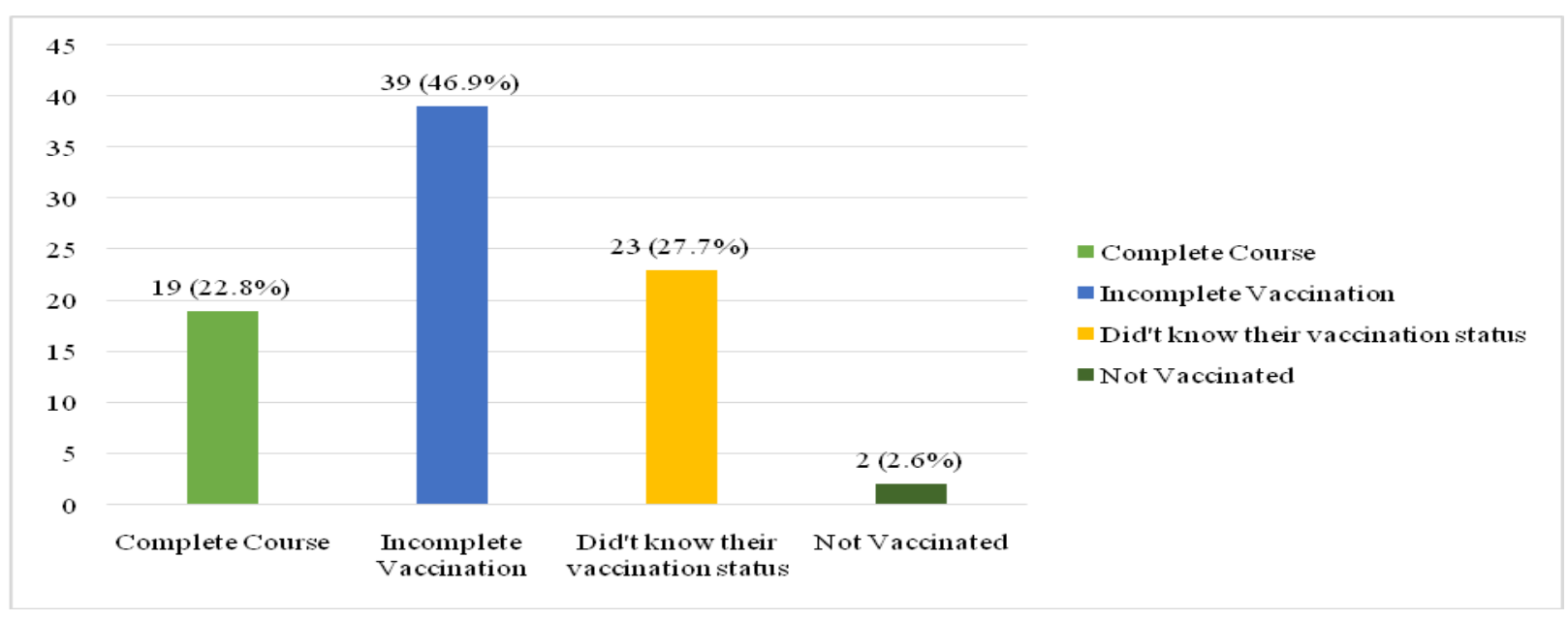




\section{Pre Exposure evaluation for Health Care Workers}

$546 \mathrm{HCWs}$ were measure for antibody to hepatitis B surface antigen (Anti-HBs)

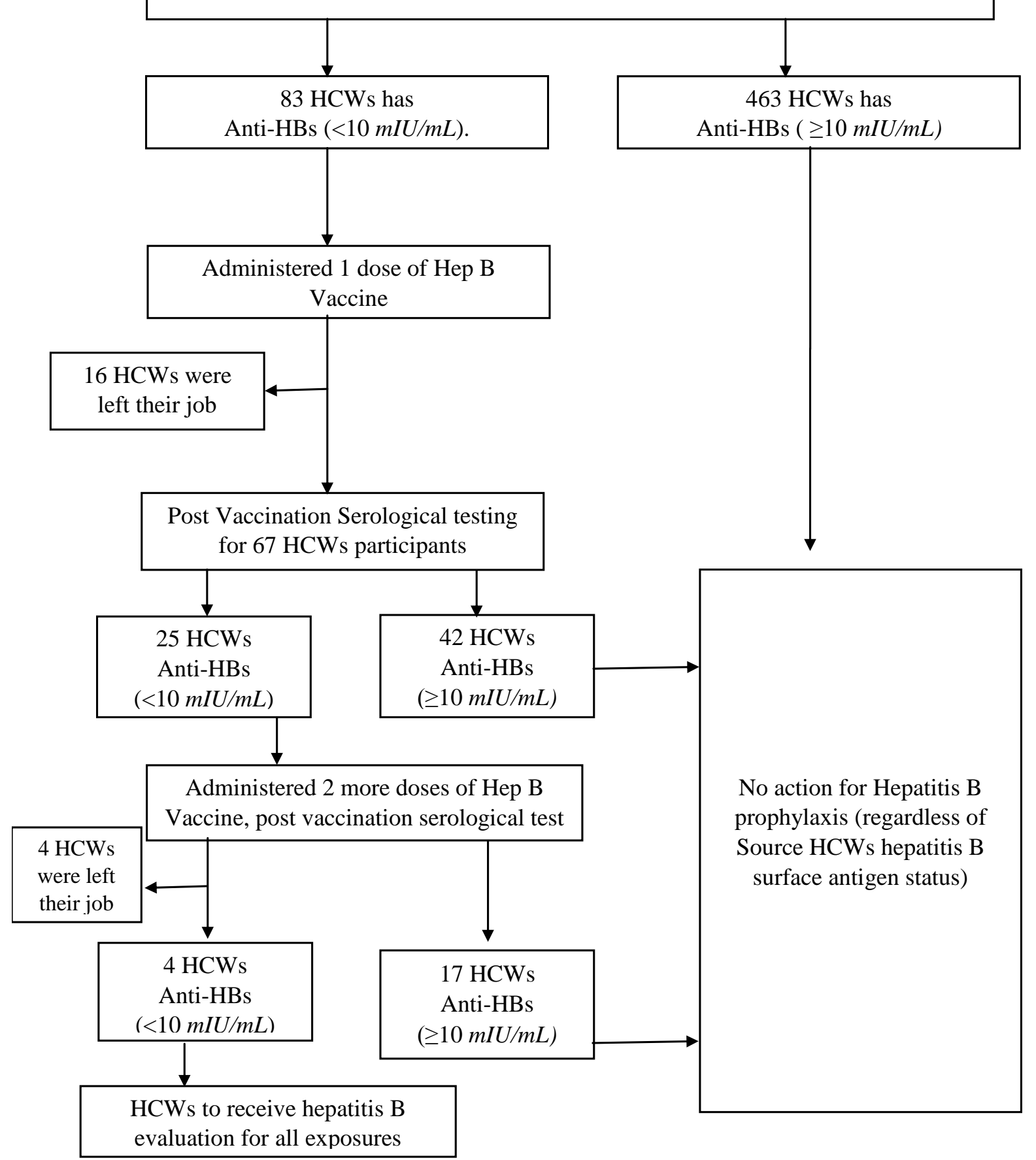

Our study evidences that the HBV vaccine efficacy and immune response to a booster vaccine indicates an extended-lasting amnestic reaction in in those who has less or nil exposure to HBI. In Health care workers, Hep B vaccine as a full course of vaccination affords long-term protection against HBI and booster vaccination does not appear to be vital 
in HCWs (10). As per the previous researchers suggestion Serological testing should be done after pre vaccination and post vaccination also to all Health Care Workers (11).

HCW who had been non-responsive in spite of repeating a complete course of vaccination had to research on their HLA allele in advance know if it is related to nonresponsiveness to the vaccine (12).

\section{Acknowledgement}

I would therefore like to offer my sincere thanks to Dr. K V Leela, M.D., Professor and Head of Microbiology for the continuous and unwavering guidance, tolerance. This would have not been possible without Mr. R. Sujith, Department of Microbiology. Motivation, support and encouragement. I consider myself fortunate to have him as my guide. I wish to express my sincere gratitude to all my Faculties Department of Microbiology for their enthusiasm, valuable advices and support given to me. I am extremely thankful to my parents Mr. Venkatesan M, Mrs. Jayanthi V, all my well-wishers and my loved one for their constant support, encouragement, guidance and prayers throughout my life.

\section{References}

1. What is hepatitis? World Health Organization, 2019. https://www.who.int/features/qa/76/en/.

2. Goel V, Kumar D, Lingaiah R, Singh S. Occurrence of Needlestick and Injuries among Health-care Workers of a Tertiary Care Teaching Hospital in North India. Journal of Laboratory Physicians 2017;9:020-5. doi:10.4103/09742727.187917.
3. Hepatitis B. World Health Organization. https://www.who.int/news-room/factsheets/detail/hepatitis-b

4. MoHFW, WHO, ILBS. Technical Consultation: World Hepatitis Day . New Delhi : s.n., 2014

5. Prevention of Hepatitis B Virus Infection in the United States: Recommendations of the Advisory Committee on Immunization Practices. Centers for Disease Control and Prevention 2018. https://www.cdc.gov/ mmwr/volumes/67/rr/rr6701a1.html

6. Vaccine Non-Responders. Hepatitis B Foundation | Baruch S Blumberg Institute. https://www.hepb.org/prevention-anddiagnosis/vaccination/vaccine-nonresponders/.

7. Euro.who.int

http://www.euro.who.int/_data/assets/pdf _file/0005/268790/WHO-guidelines-ondrawing-blood-best-practices-inphlebotomy-Eng.pdf?ua-1

8. Radia A, Amina H, Raihan B, Hajar S, Nabila S. Hepatitis B Screening and Evaluation of Hepatitis B Vaccination Status Among Medical Staff at Med VI University Hospital of Marrakech (CHU). American Journal of Laboratory Medicine 2019;4:74. doi:10.11648/j.ajlm.20190404.12.

9. Avileli S, Basireddy $\mathrm{P}$, Beldono $\mathrm{N}$, Gundela S. Evaluation of immune response to hepatitis $B$ vaccine in healthcare workers at a tertiary care hospital. Indian Journal of Medical Microbiology 2018;36:397. doi:10.4103/ijmm.ijmm_17_431.

10. Hepatitis B and the health care worker. Ask the Experts about Hepatitis B Vaccines - CDC Experts Answer Q\&As. https://www.immunize.org/askexperts/exp erts_hepb.asp (accessed March 14, 2020).

11. CDC Guidance for Evaluating HealthCare Personnel for Hepatitis B Virus Protection and for Administering Post exposure Management [Internet]. Centers 
for Disease Control and Prevention. Centers for Disease Control and Prevention; [cited 2020Mar14]. Available from:

https://www.cdc.gov/mmwr/preview/mm wrhtml/rr6210a1.htm

12. Albayrak A, Ertek M, Tasyaran MA, Pirim I. Role of HLA allele polymorphism in chronic hepatitis $B$ virus infection and HBV vaccine sensitivity in patients from eastern Turkey. Biochemical Genetics 2011.

https://www.ncbi.nlm.nih.gov/pubmed/21 188498

\section{How to cite this article:}

Balamurali, V., R. Sujith, K. V. Leela, A. Karthik and Jayaprakash, T. 2020. Prevalence of Non-responders among Medical Laboratory Technicians and Staff Nurses Evaluated for Anti Hepatitis B Surface Antigen Titre. Int.J.Curr.Microbiol.App.Sci. 9(04): 266-272. doi: https://doi.org/10.20546/ijcmas.2020.904.032 